\title{
Myocardial blood flow measures using cardiac positron emission tomography: Software comparisons
}

\author{
Navkaranbir S. Bajaj, MD, MPH, ${ }^{\mathrm{a}, \mathrm{b}, \mathrm{c}}$ Fadi G. Hage, MD, ${ }^{\mathrm{a}, \mathrm{c}}$ Jonathan McConathy, \\ $M D, P h D,{ }^{b}$ and Pradeep Bhambhvani, $M^{b}$ \\ a Division of Cardiovascular Disease, University of Alabama at Birmingham, Birmingham, AL \\ b Division of Molecular Imaging and Therapeutics, Department of Radiology, University of \\ Alabama at Birmingham, Birmingham, AL \\ c Section of Cardiology, Birmingham Veterans Affairs Medical Center, Birmingham, AL
}

Received Oct 16, 2018; accepted Oct 17, 2018

doi: $10.1007 / \mathrm{s} 12350-018-01525-7$

See related article, pp. 2007-2012

Assessment of myocardial perfusion under stress and rest conditions using various imaging modalities is the mainstay in the workup of coronary artery disease. Traditionally, the presence of relative regional perfusion defects has been the central concept behind myocardial perfusion imaging. Regional perfusion analysis is imperfect due to its relative nature, because the presence, severity, and extent of perfusion defects do not account for possible differences in absolute myocardial blood flow (MBF) at rest or stress. For example, if absolute flow is impaired globally (e.g., triple vessel disease), a comparison of the best and worst perfused regions will underestimate the severity of flow-limiting disease. Hence, information about absolute MBF and myocardial flow reserve (MFR) is desirable. ${ }^{1}$ Single-photon emission computerized tomography-myocardial perfusion imaging (SPECT-MPI) has traditionally been the workhorse for relative perfusion imaging, but several studies have shown that relative perfusion imaging may underestimate extent of disease. ${ }^{2,3}$ Furthermore, the ${ }^{99 m} \mathrm{Tc}$ flow tracers available for SPECT-MPI are limited by a relatively low first-pass extraction fraction at high flow rates, thus

Reprint requests: Pradeep Bhambhvani, MD, Division of Molecular Imaging and Therapeutics, Department of Radiology, University of Alabama at Birmingham, 619 19th Street South, JT 777, Birmingham, AL 35249; pbhambhvani@uabmc.edu

J Nucl Cardiol 2019;26:2013-7.

$1071-3581 / \$ 34.00$

Copyright (C) 2018 American Society of Nuclear Cardiology. limiting the precision and accuracy of these tracers for estimation of myocardial perfusion especially during stress. $^{4}$

With the advent and increasing availability of cardiac positron emission tomography (PET), quantification of absolute MBF and MFR has been shown to be accurate and simplified. Several studies have also shown that these measures are of diagnostic and prognostic significance beyond relative perfusion imaging. ${ }^{5-7}$ These data have resulted in a paradigm change whereby incorporation of absolute MBF and MFR measures to the relative perfusion data helps better phenotyping of flow abnormalities in patients leading to improved risk stratification. ${ }^{8}$

Although very appealing in concept the measurement of the myocardial blood flow involves several different steps all of which can potentially lead to variability in MBF and MFR measurements apart from true variability. Figure 1 highlights the potential sources of variability in measurement of MBF.

1. Patient characteristics: Patients with poor IV access may have a delayed rise or double peaked input curve, patient motion may lead to misalignment attenuation artifacts, and large myocardial infarction may preclude determination of accurate MBF measurements. ${ }^{9}$

2. Radiotracer: Four radiotracers have been most commonly used; ${ }^{82} \mathrm{Rb}$-chloride and ${ }^{13} \mathrm{~N}$-ammonia are FDA approved, whereas ${ }^{15} \mathrm{O}$-water and ${ }^{18} \mathrm{~F}$-flurpiridaz are investigational agents. The flow characteristics, i.e., retention and washout, for these tracers vary with MBF and may lead to variability in measurements. ${ }^{10-14}$

3. Scanner: Newer scanners are 3-dimensional (3D), as opposed to the older 2-dimensional (2D) (or 2D/3D) 


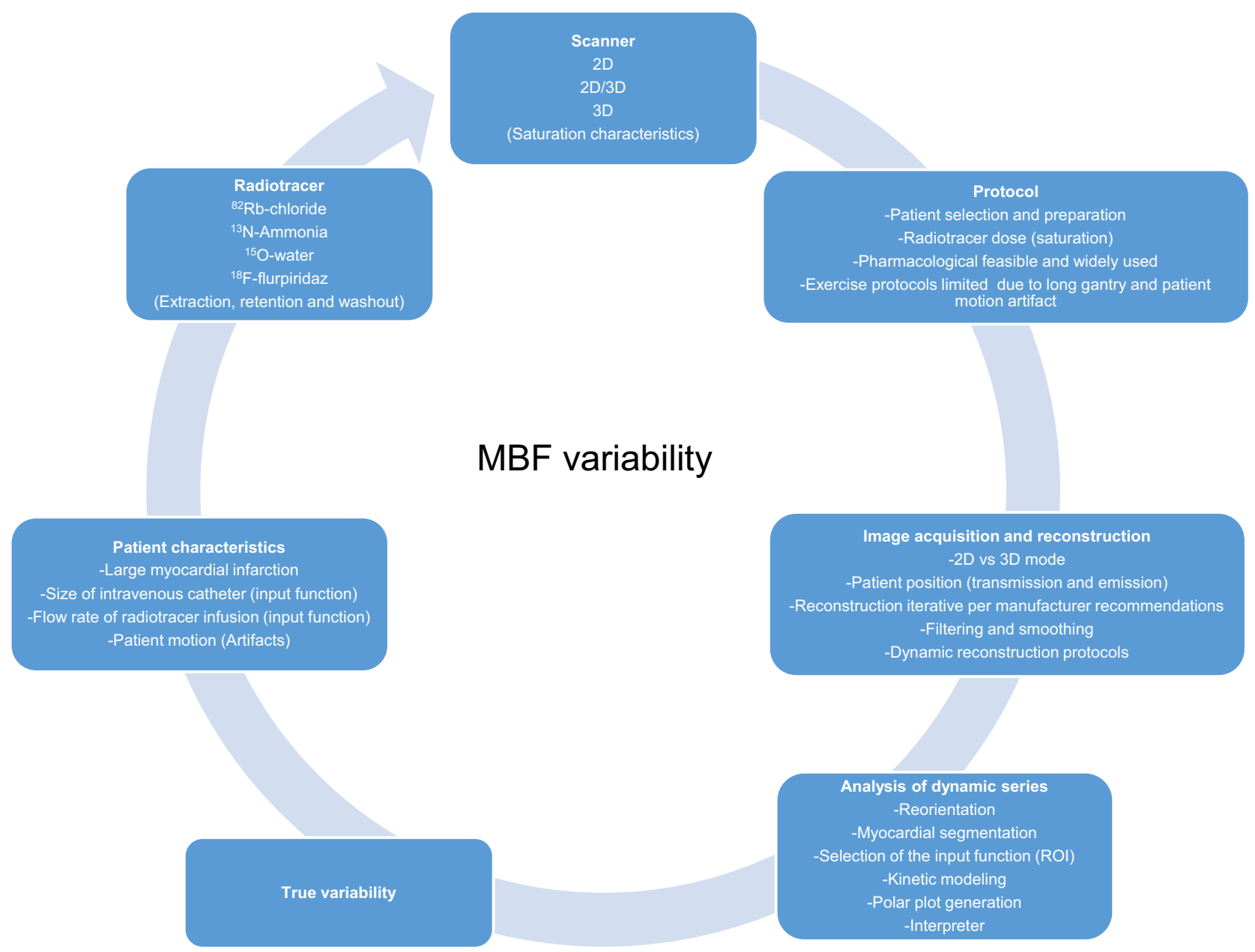

Figure 1. Factors that may lead to variability in myocardial blood flow (MBF).

systems. For 3D acquisitions, the doses of radiotracers used for 2D scanners may lead to oversaturation resulting in falsely elevated MBF. ${ }^{9}$ Scanner performance (2D vs 3D should considered) at sites with more than one scanner may also lead to variability in MBF on serial scans.

4. Protocols: Patient should be prepared for imaging in a standardized fashion. Differences in smoking, caffeine and cardiac medications may lead to variability of flows in repeated scans. Higher patient weight is associated with excessive attenuation and may lead to variability in MBF if radiotracer doses are not adjusted accordingly.

5. Image acquisition and reconstruction: $2 \mathrm{D}$ and $3 \mathrm{D}$ systems have different dead time losses, random rates which dictate the dynamic acquisition protocols. Variability in reconstruction of dynamic series, application of filters and smoothing protocols can introduce variability in MBF measurements. ${ }^{9}$
6. Analysis of dynamic series: There are 10 different software packages available for analysis of MBF from dynamic series. ${ }^{15}$ All the 10 packages have implemented variations of a one-tissue-compartment model. The image analysis process in all them consists of image reorientation, segmentation of both left ventricular myocardium and cavity and tracer kinetic modeling with variable degree of automation. ${ }^{15}$ The variability in myocardial segmentation and polar map sampling, nonuniform definition of vascular territories, dynamic motion correction, LV input region of interest, and manual interactions across software packages while processing these images has a potential to introduce variability in the MBF measures. ${ }^{16}$ Furthermore, the interpreter may be another source of variability while analyzing these series. 


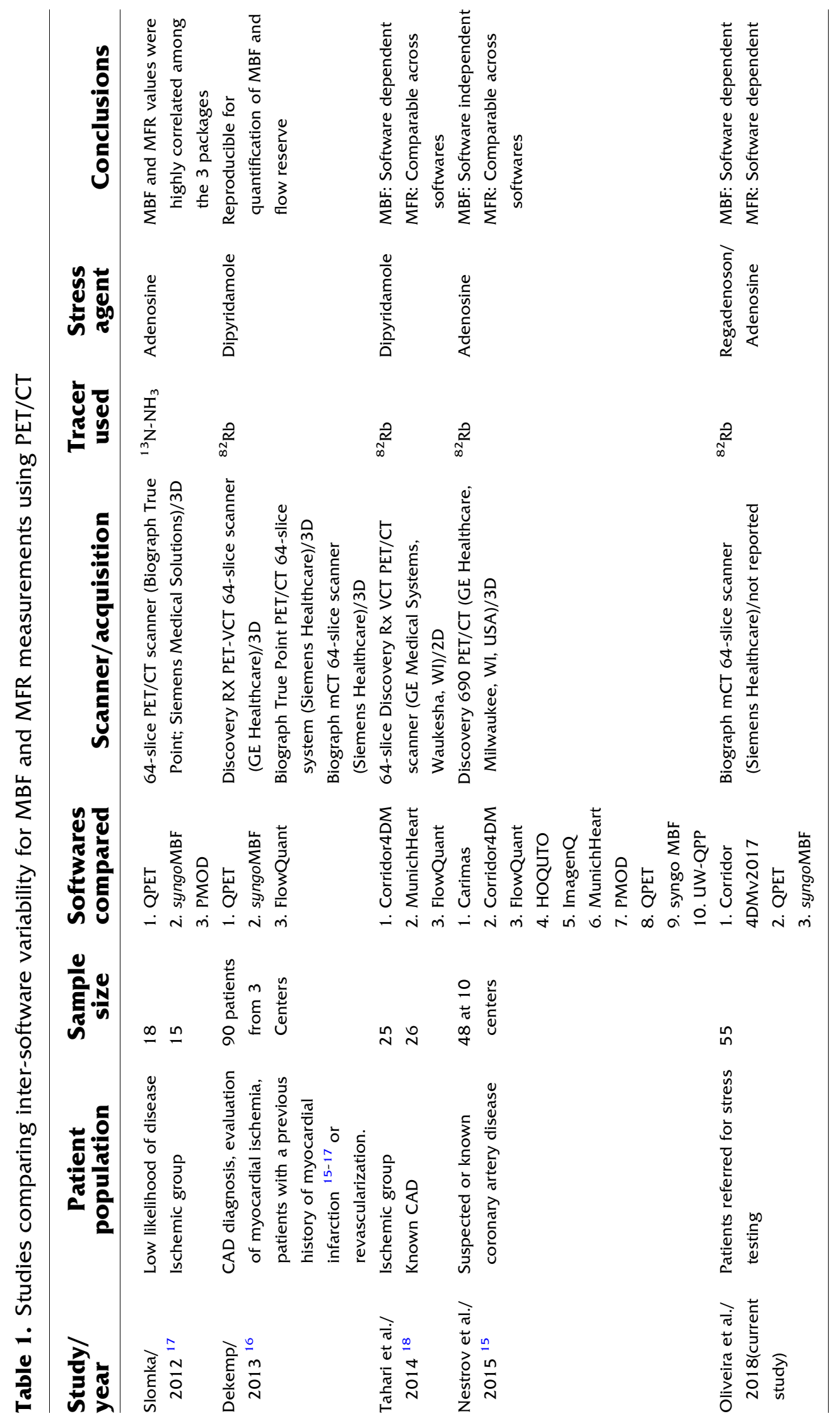


7. True variability: MBF is dynamic physiological measure and changes in disease states even when above factors are constant.

In this issue of the Journal, the study by Oliveira et al. focuses on the impact of three software packages for $\mathrm{MBF}$ ascertainment using ${ }^{82} \mathrm{Rb}$ dynamic perfusion images and conclude that MBF and MFR values are software dependent. This result is in contrast to previous studies $^{15-18}$ which have reported that MFR measures are comparable across software packages with some variation in absolute MBF measurements (Table 1). The authors attribute the inter-software variability in MBF and MFR to automated methods of finding and segmenting the myocardial and blood pools, differences in LV input region of interest (ROI), and variation introduced by manual intervention for strong subdiaphragmatic uptake across software packages.

As highlighted in Figure 1, there are several sources which can lead to variability in MBF measures. Most of the factors which can lead to variability can be dealt with by careful patient selection, standardization of protocols and operator experience. The exception to above is variability in analytic algorithms across software packages. Similar variability in software analysis of relative myocardial perfusion has been described. ${ }^{19-21}$ One should expect different algorithms to produce somewhat different quantitative results for MBF values. But MFR which is a ratio of stress over rest MBFs is thought, at least in principle, to partially correct for MBF variability assessment. Global MFR was consistently correlated across previous studies (Table 1) assessing inter-software variability and even in the current study by Oliveira et al. When a MFR threshold of 2.0 was used for defining abnormal in the current study, there were systematic classification differences such that Corridor4DMv2017 classified more patients as abnormal as compared to syngoPET and QPET. The current study brings out an interesting observation but is limited by small sample size and study design. The dynamic series in the current study were processed by a single observer who was blinded to clinical information but not to type of software or the perfusion images which could have influenced the manual interaction (input function ROI size and motion correction) with different software packages leading to systematic bias in measurement of MBF. Another limitation of this study is the lack of comparison of the software packages in terms of diagnostic accuracy according to an external reference standard such as coronary angiography derived blood flow measures. Hence, conclusions as to which software package provides the highest diagnostic accuracy cannot be derived. Future studies with much larger sample size with more precisely defined and known cardiovascular risk factors among patients and standardized protocols with comparison to a reference gold standard are needed to answer the diagnostic accuracy of software packages.

We are of the opinion that since the quantitative measurements are software specific, it may be more important to verify that repeated applications of a software package to the same dynamic series by different users produce similar results. In addition, identification of systematic bias across software may facilitate the introduction of inter-software correction factors. But while such an exercise is conducted, it is important that the appropriate risk stratification and prognostic thresholds for the individual software be used. Also while reporting serial MBF and MFR measurements, any change in the software package used between examinations should be reported to improve comparability.

\section{PRACTICAL CONCLUSION}

Careful patient selection, standardization of protocols, use of same camera and software for serial studies, knowledge of software-specific thresholds for diagnostic and prognostic significance and experience of operator may help reduce variability of MBF measurements. Larger studies are needed to better understand the nuances related with different software packages for analysis of dynamic series. Quantitative absolute MBF measurements may not be as reliable and reproducible as other key measures in medicine, such as blood counts or metabolic profiles, but when interpreted by experienced operators using standard protocols and same software these novel measurements do add value to relative flow assessment and provide powerful risk prognostication data.

\section{Disclosure}

Dr. Bajaj is supported by American College of Cardiology Presidential Career Development Award. Dr. Hage has received grants from Astellas Pharma, Novartis Pharma, GE healthcare. Jonathan McConathy and Pradeep Bhambhvani have no relevant disclsoures.

\section{References}

1. Bengel FM. Leaving relativity behind: quantitative clinical perfusion imaging. J Am Coll Cardiol 2011;58:749-51.

2. Lima RS, Watson DD, Goode AR, et al. Incremental value of combined perfusion and function over perfusion alone by gated SPECT myocardial perfusion imaging for detection of severe three-vessel coronary artery disease. J Am Coll Cardiol 2003;42:64-70 
3. Berman DS, Kang X, Slomka PJ, et al. Underestimation of extent of ischemia by gated SPECT myocardial perfusion imaging in patients with left main coronary artery disease. J Nucl Cardiol 2007;14:521-8.

4. Watson DD, Glover DK. Overview of tracer kinetics and cellular mechanisms of uptake. Clinical Nuclear Cardiology. 4th ed. Philadelphia: Mosby; 2010. p. 3-13.

5. Bajaj NS, Osborne MT, Gupta A, et al. Coronary Microvascular Dysfunction and Cardiovascular Risk in Obese Patients. J Am Coll Cardiol 2018;72:707-17.

6. Osborne MT, Bajaj NS, Taqueti VR, et al. Coronary microvascular dysfunction identifies patients at high risk of adverse events across cardiometabolic diseases. J Am Coll Cardiol 2017;70:2835-7.

7. Bravo PE, Bergmark BA, Vita T, et al. Diagnostic and prognostic value of myocardial blood flow quantification as non-invasive indicator of cardiac allograft vasculopathy. Eur Heart J 2018;39:316-23.

8. Gupta A, Taqueti VR, van de Hoef TP, et al. Integrated Noninvasive Physiological Assessment of Coronary Circulatory Function and Impact on Cardiovascular Mortality in Patients With Stable Coronary Artery Disease. Circulation 2017;136:2325-36.

9. Murthy VL, Bateman TM, Beanlands RS, et al. Clinical quantification of myocardial blood flow using PET: Joint position paper of the SNMMI cardiovascular council and the ASNC. J Nucl Med 2018;59:273-93.

10. Sherif HM, Nekolla SG, Saraste A, et al. Simplified quantification of myocardial flow reserve with flurpiridaz F 18: Validation with microspheres in a pig model. J Nucl Med 2011;52:617-24.

11. Lortie M, Beanlands RS, Yoshinaga K, Klein R, Dasilva JN, DeKemp RA. Quantification of myocardial blood flow with 82Rb dynamic PET imaging. Eur J Nucl Med Mol Imaging 2007;34:1765-74.

12. Renaud JM, DaSilva JN, Beanlands RS, DeKemp RA. Characterizing the normal range of myocardial blood flow with (8)(2)rubidium and (1)(3)N-ammonia PET imaging. J Nucl Cardiol 2013;20:578-91.
13. Nienaber CA, Ratib O, Gambhir SS, et al. A quantitative index of regional blood flow in canine myocardium derived noninvasively with $\mathrm{N}-13$ ammonia and dynamic positron emission tomography. $\mathrm{J}$ Am Coll Cardiol 1991;17:260-9.

14. Schelbert HR, Phelps ME, Hoffman E, Huang SC, Kuhl DE. Regional myocardial blood flow, metabolism and function assessed noninvasively with positron emission tomography. Am J Cardiol 1980;46:1269-77.

15. Nesterov SV, Deshayes E, Sciagrà R, et al. Quantification of Myocardial Blood Flow in Absolute Terms u27sing (82)Rb PET Imaging: Results of RUBY-10 - a multicenter study comparing ten computer analysis programs. JACC Cardiovasc imaging 2014;7:1119-27.

16. Dekemp RA, Declerck J, Klein R, et al. Multisoftware reproducibility study of stress and rest myocardial blood flow assessed with 3D dynamic PET/CT and a 1-tissue-compartment model of 82Rb kinetics. J Nucl Med 2013;54:571-7.

17. Slomka PJ, Alexanderson E, Jacome R, et al. Comparison of clinical tools for measurements of regional stress and rest myocardial blood flow assessed with $13 \mathrm{~N}$-ammonia PET/CT. J Nucl Med 2012;53:171-81.

18. Tahari AK, Lee A, Rajaram M, et al. Absolute myocardial flow quantification with (82)Rb PET/CT: Comparison of different software packages and methods. Eur J Nucl Med Mol Imaging 2014;41:126-35.

19. Ather S, Iskandrian AE, Hage FG. Sources of variability in the measurement of perfusion defect size using commercially available software programs: Are there gender differences? J Nucl Cardiol 2017;24:1089-93.

20. Ather S, Iqbal F, Gulotta J, et al. Comparison of three commercially available softwares for measuring left ventricular perfusion and function by gated SPECT myocardial perfusion imaging. J Nucl Cardiol 2014;21:673-81.

21. Alexiou S, Georgoulias P, Angelidis G, et al. Myocardial perfusion and left ventricular quantitative parameters obtained using gated myocardial SPECT: Comparison of three software packages. J Nucl Cardiol 2018;25:911-24. 